\title{
The flow angle beneath the gastrojejunostomy predicts delayed gastric emptying in Roux-en-Y reconstruction after distal gastrectomy
}

\author{
Toshihiko Masui · Toyonari Kubora $\cdot$ Yasutaka Nakanishi $\cdot$ Keiko Aoki $\cdot$ Shinichi Sugimoto \\ Michio Takamura $\cdot$ Hiroshi Takeda $\cdot$ Koji Hashimoto $\cdot$ Atsuo Tokuka
}

Received: 13 April $2011 /$ Accepted: 23 September 2011/Published online: 2 November 2011

(C) The International Gastric Cancer Association and The Japanese Gastric Cancer Association 2011

\begin{abstract}
Background Delayed gastric emptying without mechanical obstruction after Roux-en-Y reconstruction has been defined as Roux stasis syndrome. It occurs in $10-30 \%$ of patients after such reconstruction. So far, the cause of this stasis has not been completely identified. This study aimed to reduce Roux stasis using surgical techniques.

Methods From November 2007 to October 2010, we performed 101 distal gastrectomies with Roux-en-Y reconstruction. All the gastrojejunostomies were performed with end-to-end anastomoses. Roux stasis was analyzed with respect to tumor location, extent of the dissection, tumor progression, operation time, antecolic/retrocolic reconstruction, and the shape of the gastrojejunostomy. The shape of the gastrojejunostomy was evaluated by contrast gastroradiography 4 days after the operation.

Results Roux stasis syndrome was observed in 17 of the 101 patients. There was no relationship between the extent of the dissection, tumor progression, or operation time and the occurrence of Roux stasis. There was no difference in the incidence of Roux stasis between antecolic and retrocolic reconstructions. However, the group that displayed a straight anastomotic shape on contrast radiography demonstrated an apparently lower incidence of Roux stasis $(p=0.0003)$. In addition, Roux-en-Y reconstruction following gastric cancer was more frequently followed by Roux stasis in the antrum than in the midstomach $(p=0.0036)$. Cases of Roux stasis occurred 11.8 days
\end{abstract}

T. Masui ( $)$ · T. Kubora · Y. Nakanishi · K. Aoki ·

S. Sugimoto $\cdot$ M. Takamura $\cdot$ H. Takeda $\cdot$ K. Hashimoto $\cdot$

A. Tokuka

Department of Surgery, Shimane Prefectural Central Hospital,

4-1-1 Himebara, Izumo, Shimane 693-8555, Japan

e-mail: tmasui@kuhp.kyoto-u.ac.jp after surgery on average and resolved within 2 weeks on average.

Conclusions Our findings demonstrate the substantial benefits of a straight anastomosis of the gastrojejunostomy for the prevention of Roux stasis syndrome.

Keywords Roux stasis syndrome $\cdot$ Delayed gastric emptying · Distal gastrectomy

\section{Introduction}

Roux-en-Y reconstruction has several advantages compared to other reconstruction methods utilized following distal gastrectomy. Roux-en-Y reconstruction results in a lower frequency of remnant or reflux gastritis $[1,2]$ and a lower possibility of remnant gastric cancer [3], and anastomotic leakage is rare. In addition, a Roux-en- $Y$ anastomosis potentially prevents obstruction of the dietary route caused by recurrence around the pylorus and has a lower possibility of anastomotic leakage than other reconstruction methods [4]. Conversely, delayed gastric emptying tends to occur more frequently in Roux-en-Y reconstruction than in Billroth I reconstruction [5, 6].

Delayed gastric emptying without mechanical obstruction after Roux-en-Y reconstruction has been defined as Roux stasis syndrome (Roux stasis) and occurs at frequencies of $10-30 \%$ after Roux-en-Y reconstruction [710]. It is diagnosed on the basis of clinical symptoms such as epigastric fullness, postprandial pain, nausea, and vomiting. Once Roux stasis occurs, the hospital stay is prolonged, and the patient's quality of life is reduced.

There have been many causes proposed for the development of Roux stasis, including functional obstruction of the Roux limb, an ectopic pacemaker arising in the Roux 
limb that drives contractions in a reverse direction toward the stomach [11, 12], and gastroparesis after vagotomy [13]. On the basis of these hypotheses, uncut Roux Y reconstruction [14] or rho-shaped Roux Y reconstruction have been proposed and found to be effective in avoiding Roux stasis [15, 16]. End-to-end anastomosis of the gastrojejunostomy has been reported to reduce Roux stasis compared to the incidence observed with end-to-side anastomosis [17]. However, to date, a practical method to reduce Roux stasis has not been definitely identified. Here, we report on our investigation of the shape of the gastrojejunostomy and its relation to Roux stasis. The aim of this study was to develop a method to reduce Roux stasis using surgical techniques.

\section{Patients and methods}

From November 2007 to October 2010, we performed 101 gastrectomies in patients diagnosed with gastric cancer and performed reconstruction with Roux-en-Y anastomoses. All patients had tumors located in the lower two-thirds of the stomach and were treated by distal or subtotal gastrectomy. D1 or D2 lymphadenectomy was defined on the basis of the Japanese classification for standard dissection. All of the gastrojejunostomies were performed with an end-to-end anastomosis with a 1-layer Gambee intermittent suture (3-0 braided filaments). The jejunum was divided at $20 \mathrm{~cm}$ distal to the Treitz ligament, and the oral portion of the jejunum was anastomosed to the mid-jejunum at $30 \mathrm{~cm}$ distal to the gastrojejunostomy. The anastomotic size was adjusted to the caliber of the jejunum. We performed fixation of the remnant stomach to the transverse mesocolon in retrocolic anastomosis and no fixation was done in antecolic anastomosis. From 2007 to 2008, we performed retrocolic reconstruction, and from mid-2008 to 2010, our reconstruction method was changed to the antecolic method due to reports of reduced Roux stasis with the antecolic method [18].

We performed contrast gastrography on postoperative day (POD) 4 to verify the absence of anastomotic leakage and the passage of the medium. When the gastrography revealed no leakage, patients began eating.

We defined Roux stasis as follows: (1) the presence of symptoms such as nausea, vomiting, or abdominal fullness; and (2) starting a solid diet after POD 7 (which is 1 day later than the average date for solid oral intake), or refasting. We defined "mild" Roux stasis as less than 10 days of continuous fasting after the operation, or 3 days of refasting; "severe" as more than 17 days of continuous fasting after the operation, or 10 days of refasting; and other conditions as "moderate." All patients with symptoms of delayed gastric emptying were examined to exclude mechanical obstruction, using a gastrointestinal scope, from POD 10 to POD 21 when the anastomosis was sufficiently strong for endoscopic examination. The state of fasting/refasting was decided by the doctors in charge of patients in the round every morning. The doctors initially started the patients on cisapride for nausea or vomiting, and if the symptoms remained, the patient's oral intake was stopped until the nausea or vomiting vanished.

Clinical data such as age, sex, tumor location, dissection, T stage, operation time, blood loss, the direction of the gastrojejunostomy as estimated by contrast gastrography, and the incidence of Roux stasis were analyzed. We categorized gastrojejunostomy direction into 3 groups: type I, gastrojejunostomy direction being more than $30^{\circ}$ to the duodenal side; type III, gastrojejunostomy direction being more than $30^{\circ}$ to the spleen side; and type II, all other gastrojejunostomy direction angles (Fig. 1). The direction of the Roux limbs was assessed by the flow direction of the contrast medium passed between the anastomosis and the jejunum $1 \mathrm{~cm}$ below the anastomosis. The direction angles were evaluated against the patients' vertebrae. The anastomotic shape was estimated in the anterior-to-posterior direction by gastrography. We omitted the anterior or posterior bend, as we found this extremely difficult to estimate with contrast gastrography. Qualitative data were compared using the $\chi^{2}$ test and Fisher's exact test, and the Kruskal-Wallis test was utilized when necessary. All statistical analysis was performed with JMP version 6.0 (Cary, NC, USA), and a probability of less than 0.05 was deemed significant.

\section{Results}

The overall incidence of Roux stasis syndrome was $17 \%$ (Table 1). No postoperative hospital deaths occurred in this study. There were no significant differences in sex, age,
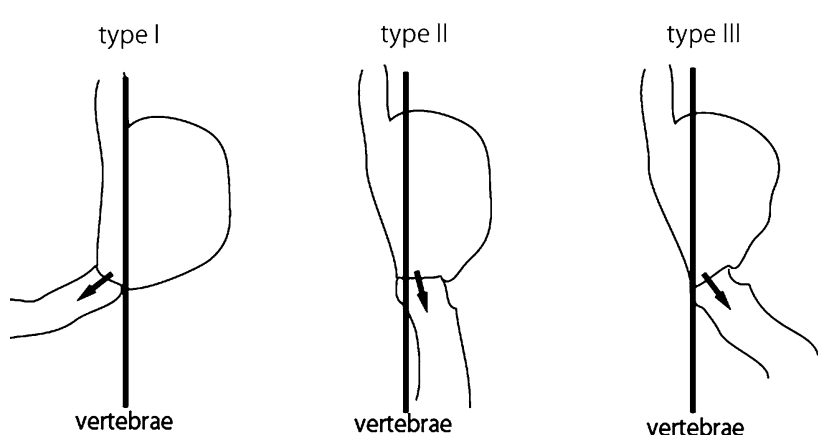

Fig. 1 Classification of gastrojejunostomy. Type I gastrojejunostomy direction being more than $30^{\circ}$ to the duodenal side, type III gastrojejunostomy direction being more than $30^{\circ}$ to the spleen side, type II all other gastrojejunostomy direction angles 
Table 1 Association of clinical and surgical factors with Roux stasis syndrome
$R S$ Roux stasis syndrome, $N A$ not applicable, $\mathrm{m} / \mathrm{sm} \mathrm{mp} A \mathrm{M}$

\begin{tabular}{|c|c|c|c|c|}
\hline & $\mathrm{RS}(-)$ & RS (+) & Total & $p$ \\
\hline Number of patients & 84 & 17 & 101 & NA \\
\hline \multicolumn{5}{|l|}{ Sex } \\
\hline Male & 55 & 14 & 69 & \\
\hline Female & 29 & 3 & 32 & 0.2543 \\
\hline \multicolumn{5}{|l|}{ Age (years) } \\
\hline Mean (min-max) & $70.2(45-89)$ & $67.4(46-82)$ & $69.7(45-89)$ & 0.2894 \\
\hline \multicolumn{5}{|l|}{ Operation time (min) } \\
\hline Mean (min-max) & $269.9(125-503)$ & $269.2(133-406)$ & $269.8(125-503)$ & 0.7199 \\
\hline \multicolumn{5}{|l|}{ Blood loss (g) } \\
\hline Mean (min-max) & $387.5(22-1257)$ & $369.3(69-1201)$ & $384.6(22-1257)$ & 0.8154 \\
\hline \multicolumn{5}{|l|}{ Tumor depth } \\
\hline $\mathrm{m} / \mathrm{sm}$ & 40 & 10 & 50 & \\
\hline$>\mathrm{mp}$ & 43 & 8 & 51 & 0.7566 \\
\hline \multicolumn{5}{|l|}{ Tumor location } \\
\hline A & 37 & 12 & 49 & \\
\hline M & 47 & 5 & 52 & 0.0458 \\
\hline \multicolumn{5}{|l|}{ Antecolic/retrocolic } \\
\hline Antecolic & 50 & 13 & 63 & \\
\hline Retrocolic & 34 & 4 & 38 & 0.2734 \\
\hline \multicolumn{5}{|l|}{ Type } \\
\hline I & 18 & 11 & 29 & \\
\hline II & 62 & 6 & 68 & \\
\hline III & 4 & 0 & 4 & 0.0014 \\
\hline \multicolumn{5}{|l|}{ Type } \\
\hline I & 18 & 11 & 29 & \\
\hline II + III & 66 & 6 & 72 & 0.0003 \\
\hline
\end{tabular}

operative time, $\mathrm{T}$ stage, or blood loss in relation to Roux stasis. Roux stasis occurred in both the antecolic and retrocolic reconstruction groups. Roux-en-Y reconstruction following gastric cancer was more frequently followed by Roux stasis in the antrum than in the midstomach $(p=0.0036)$.

When we categorized the direction of the gastrojejunostomy into 3 groups according to the contrast gastrography findings, the frequency was $29 \%$ for the type I direction, $67 \%$ for type II, and $4 \%$ for type III. The type II direction demonstrated a significantly lower frequency of Roux stasis than the other types ( $p=0.0014$; Table 1) and a lower severity of Roux stasis ( $p=0.0187$; Table 2). When we categorized the patients into 2 classes by flow direction, type I and type II + III, an apparently high incidence of Roux stasis was observed in the type I group ( $p=0.0003$; Table 1 , bottom). However, there was no relationship between tumor location and the direction of the gastrojejunostomy (data not shown).

Approximately half of the 17 patients with Roux stasis were placed in the severe group (9 patients, 53\%), the moderate group consisted of 5 patients $(29 \%)$, and the mild
Table 2 Association of clinical and surgical factors with the severity of Roux stasis syndrome

\begin{tabular}{lllllll}
\hline & $\begin{array}{l}\text { RS } \\
(-)\end{array}$ & $\begin{array}{l}\text { RS } \\
(\text { mild })\end{array}$ & $\begin{array}{l}\text { RS } \\
(\text { moderate })\end{array}$ & $\begin{array}{l}\text { RS } \\
(\text { severe })\end{array}$ & Total & $p$ \\
\hline $\begin{array}{l}\text { Number of } \\
\text { patients }\end{array}$ & 84 & 3 & 5 & 9 & 101 & NA \\
$\begin{array}{l}\text { Tumor location } \\
\text { A }\end{array}$ & 37 & 1 & 4 & 7 & 49 & \\
M & 47 & 2 & 1 & 2 & 52 & 0.1107 \\
Type & & & & & & \\
I & 18 & 1 & 4 & 6 & 29 & \\
II & 62 & 2 & 1 & 3 & 68 & \\
III & 4 & 0 & 0 & 0 & 4 & 0.0187 \\
\hline
\end{tabular}

$R S$ Roux stasis syndrome

group contained 3 patients (18\%), suggesting that most cases of Roux stasis took at least 10 days to resolve.

We investigated 6 patients with type II Roux stasis in detail and found through gastrointestinal endoscopy that 4 patients had extreme paresis of the Roux limbs. The remaining 2 patients experienced refasting after they were discharged, suggesting a relatively large amount of food 
Table 3 Characteristics of patients with Roux stasis syndrome
$R S$ Roux stasis syndrome, $P O D$ postoperative day

\begin{tabular}{lllllll}
\hline $\begin{array}{l}\text { Patient } \\
\text { number }\end{array}$ & Type & RS & $\begin{array}{l}\text { Age } \\
\text { (years) }\end{array}$ & $\begin{array}{l}\text { POD } \\
\text { of RS }\end{array}$ & $\begin{array}{l}\text { Recovery } \\
\text { date (POD) }\end{array}$ & $\begin{array}{l}\text { RS period } \\
\text { (days) }\end{array}$ \\
\hline 1 & I & Mild & 66 & 14 & 16 & 2 \\
2 & I & Moderate & 53 & 13 & 20 & 7 \\
3 & I & Moderate & 66 & 21 & 30 & 9 \\
4 & I & Moderate & 76 & 11 & 20 & 5 \\
5 & I & Moderate & 80 & 13 & 18 & 14 \\
6 & I & Severe & 46 & 11 & 25 & 17 \\
7 & I & Severe & 82 & 8 & 25 & 11 \\
8 & I & Severe & 74 & 7 & 18 & 17 \\
9 & I & Severe & 69 & 8 & 25 & 12 \\
10 & I & Severe & 53 & 13 & 66 & 3 \\
11 & I & Severe & 76 & 9 & 21 & 4 \\
12 & II & Mild & 83 & 15 & 18 & 6 \\
13 & II & Mild & 53 & 14 & 18 & 14 \\
14 & II & Moderate & 70 & 11 & 17 & 13 \\
15 & II & Severe & 71 & 13 & 27 & 12.4 \\
16 & II & Severe & 66 & 11 & 24 & 22 \\
17 & II & Severe & 62 & 8 & 24.1 & \\
Average & & & & 11.8 & & \\
\hline
\end{tabular}
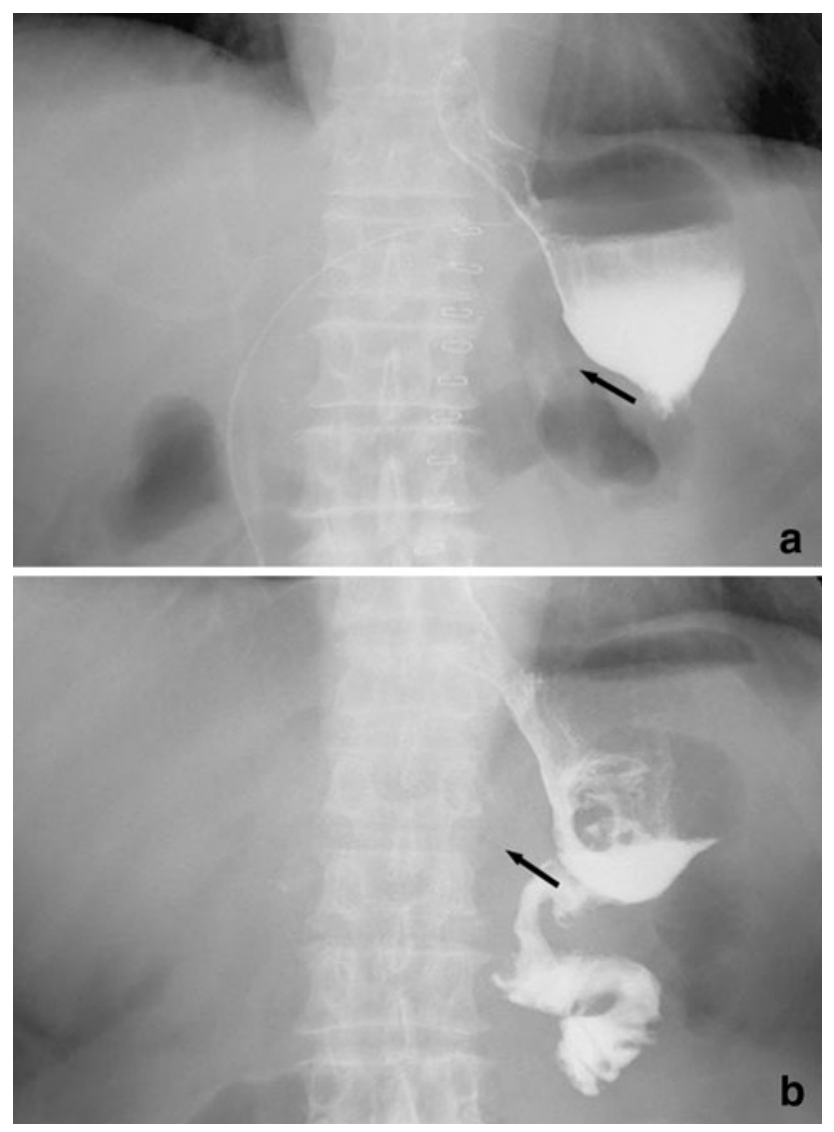

Fig. 2 Postoperative fluoroscopy image. a Postoperative fluoroscopy (postoperative day [POD] 4) revealed no passage of the contrast medium, $\mathbf{b}$ followed by improvement without operation after POD 17 (patient \#8). Arrows show the flow direction of the contrast medium intake at home. On average, Roux stasis occurred 11.8 days after the operation, and refeeding occurred approximately 24.1 days after the operation (Table 3).

Contrast gastrography demonstrated retention of the contrast medium in the remnant stomach and slow gastric evacuation (Fig. 2a), but smooth outflow was observed when the patient recovered from Roux stasis (Fig. 2b). Patient \#9 presented a strong bend of the Roux limb to the lesser curvature of the stomach (Fig. 3a), but the gastrointestinal scope revealed no stenosis (Fig. 3b).

\section{Discussion}

In the hepatobiliary-pancreatic field, the straight position of the stomach following a pylorus-preserving pancreaticoduodenectomy (PPPD) has recently been reported to reduce delayed gastric emptying $[19,20]$. In the present study, we assumed that the gravitational dietary drop into the Roux limbs might be one of the factors that prevented delayed gastric emptying regardless of stasis in the Roux limbs, and we examined the direction of the anastomosis as estimated by contrast gastrography. Although factors such as the extent of dissection, blood loss, and operation time were not significantly related to Roux stasis, the straight flow direction group exhibited an apparently lower frequency of Roux stasis. Although there were only 4 type III patients, a number which is too small for statistical analysis, the lack of Roux stasis in the type III patients suggests that bending to the duodenal side by more than $30^{\circ}$ is an important 
Fig. 3 Patient \#9 exhibited a an extreme bend of the gastrojejunostomy on fluoroscopy, but b no stenosis was observed on gastrointestinal fiberscopy

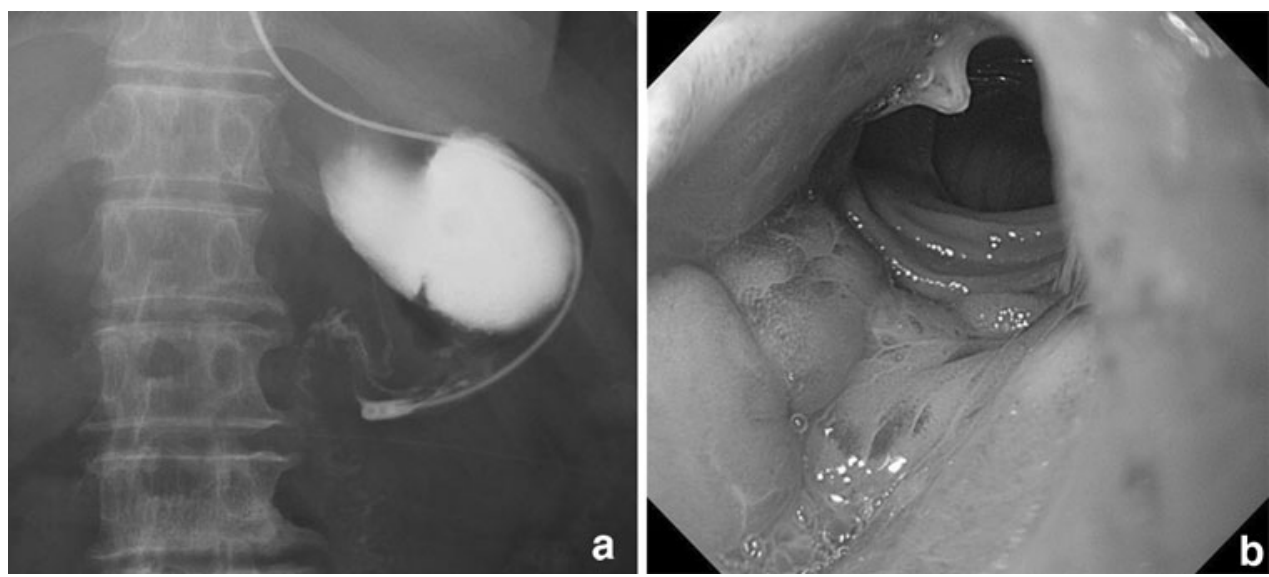

factor in causing Roux stasis. One of the reasons for this could be the suture of the remnant stomach on the duodenal side to which the Roux limb could easily adhere. Indeed, gastrography of the Roux stasis in patient \#9 (Fig. 3) showed that the Roux limb adhered to the suture.

Data from our present study suggest that tumor location might affect Roux stasis, as demonstrated in patients with tumors in the antrum who appeared to have a higher susceptibility to Roux stasis. Although we could not find direct evidence of a relationship between remnant stomach size and the tumor location, a relatively large remnant stomach has been shown to cause a high incidence of Roux stasis $[21,22]$ and thus a large remnant stomach could affect Roux stasis in patients with tumors in the antrum.

There are several methods to be considered to ensure the optimal direction of the anastomosis: (1) trimming of the greater curvature to optimize the angle of the gastrojejunostomy (Fig. 4a); (2) retaining the enasogastric tube in the Roux limb until one day after the operation (Fig. 4b); and (3) placing the Roux limb in a straight manner in the left iliac fossa, which is outside the descending colon, for smooth dietary flow (Fig. 4c). Although we did not intend to use these techniques in this study, they are potentially effective for ensuring a straight direction of the anastomosis according to the findings of PPPD studies [19, 20].

The angle of the gastrojejunostomy on POD 4 appeared to persist, at least during the Roux stasis period. We note two observations: (1) as shown in Fig. 2, contrast gastrography revealed that the angle was unchanged after the Roux stasis period, and (2) adhesion of the intestine occurred quickly, being observed 1 day after the operation, similar to a report of immediate postlaparotomy small bowel obstruction [23]. The first observation indicates that Roux stasis occurs not only because of the direction of the anastomosis but also because of the initially low peristalsis after the operation.

The direction of the anastomosis is not the only factor that affects Roux stasis. Six patients in the present study
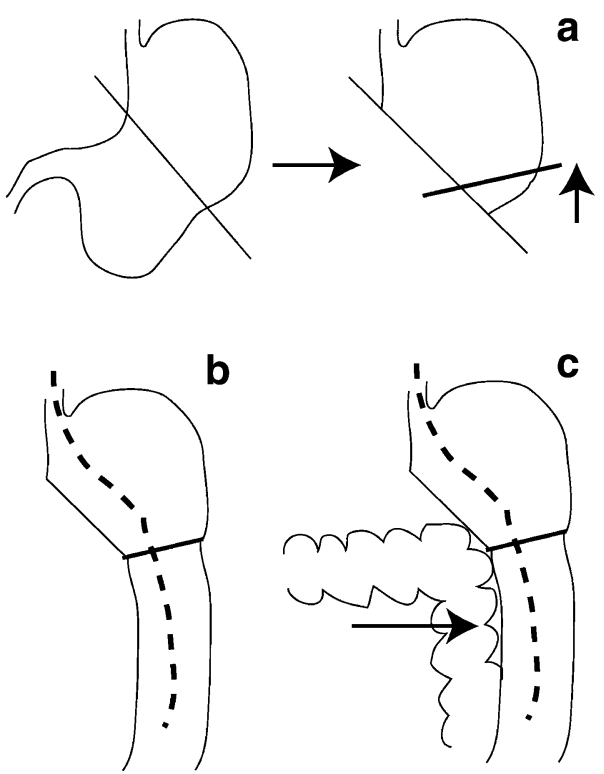

Fig. 4 Proposed technique for reducing Roux stasis syndrome. a Trimming the edges of the greater curvature widely, b retaining the nasogastric tube in the Roux limb, and c placing the Roux limb in the left iliac fossa outside the descending colon

developed Roux stasis despite having a straight anastomosis direction. Of these patients, 4 had extremely low peristalsis in the Roux limbs that could not be resolved by surgical techniques. We could not identify the contraction of the Roux limbs in the reverse fashion where ectopic pacemakers were concerned. The remaining 2 patients experienced refasting after discharge and exhibited improvement after the minimum fasting date, suggesting an imbalance between the incoming diet and contraction of the Roux limbs.

Delayed gastric emptying occurring in the early postoperative period is generally thought to resolve spontaneously within 6 weeks of surgery, and the temptation to reoperate on a nonobstructed stomach should therefore be avoided [21]. Upper gastrointestinal fiberscopy is usually used to identify stenosis or obstruction, but fiberscopy 
revealed no stenosis in our patient \#9, although a strong bend was detected on contrast gastrography, suggesting the obstruction was caused by flap formation at the bending point. This suggests that the assessment of mechanical stenosis is difficult and cannot be achieved only by gastrointestinal endoscopy. Patient \#9 spontaneously recovered from the stasis even in this situation, indicating that patients with no mechanical obstruction should not undergo reoperation.

In conclusion, Roux stasis potentially occurs after Rouxen-Y reconstruction in a certain number of patients. However, because of its severity and difficulties in analysis, reports on Roux stasis are rare. Although the present study was not randomized, nor did it have a large sample size, our findings suggest the possibility of avoiding or reducing Roux stasis by an operational technique, i.e., performing the anastomosis in an appropriate direction.

\section{References}

1. Fukuhara K, Osugi H, Takada N, Takemura M, Higashino M, Kinoshita H. Reconstructive procedure after distal gastrectomy for gastric cancer that best prevents duodenogastroesophageal reflux. World J Surg. 2002;26(12):1452-7.

2. Fukuhara K, Osugi H, Takada N, Takemura M, Ohmoto Y, Kinoshita H. Quantitative determinations of duodenogastric reflux, prevalence of Helicobacter pylori infection, and concentrations of interleukin-8. World J Surg. 2003;27(5):567-70.

3. Langhans P, Schonleben K, Bunte H. The routine use of Rouxen-Y anastomosis in gastric surgery. Scand J Gastroenterol Suppl. 1981;67:247-9.

4. Hoya Y, Mitsumori N, Yanaga K. The advantages and disadvantages of a Roux-en-Y reconstruction after a distal gastrectomy for gastric cancer. Surg Today. 2009;39(8):647-51.

5. Mathias JR, Fernandez A, Sninsky CA, Clench MH, Davis RH. Nausea, vomiting, and abdominal pain after Roux-en-Y anastomosis: motility of the jejunal limb. Gastroenterology. 1985;88(1 Pt 1):101-7.

6. Miedema BW, Kelly KA, Camilleri M, et al. Human gastric and jejunal transit and motility after Roux gastrojejunostomy. Gastroenterology. 1992;103(4):1133-43.

7. Bar-Natan M, Larson GM, Stephens G, Massey T. Delayed gastric emptying after gastric surgery. Am J Surg. 1996;172(1):24-8.

8. Britton JP, Johnston D, Ward DC, Axon AT, Barker MC. Gastric emptying and clinical outcome after Roux-en-Y diversion. Br J Surg. 1987;74(10):900-4.
9. Gustavsson S, Ilstrup DM, Morrison P, Kelly KA. Roux-Y stasis syndrome after gastrectomy. Am J Surg. 1988;155(3):490-4.

10. Herrington JL Jr, Scott HW Jr, Sawyers JL. Experience with vagotomy-antrectomy and Roux-en-Y gastrojejunostomy in surgical treatment of duodenal, gastric, and stomal ulcers. Ann Surg. 1984;199(5):590-7.

11. Hermon-Taylor J, Code CF. Localization of the duodenal pacemaker and its role in the organization of duodenal myoelectric activity. Gut. 1971;12(1):40-7.

12. van der Mijle HC, Kleibeuker JH, Limburg AJ, Bleichrodt RP, Beekhuis H, van Schilfgaarde R. Manometric and scintigraphic studies of the relation between motility disturbances in the Roux limb and the Roux-en-Y syndrome. Am J Surg. 1993;166(1): $11-7$.

13. Mathias JR, Khanna R, Nealon WH, Browne RM, Reeves-Darby VG, Clench MH. Roux-limb motility after total gastrectomy and Roux-en-Y anastomosis in patients with Zollinger-Ellison syndrome. Dig Dis Sci. 1992;37(4):545-50.

14. Miedema BW, Kelly KA. The Roux stasis syndrome. Treatment by pacing and prevention by use of an 'uncut' Roux limb. Arch Surg. 1992;127(3):295-300.

15. Ou-Uti K, Sugiyama Y, Hada R. rho-Shaped anastomosis: a reconstruction of the alimentary tract after total gastrectomy. Am J Surg. 1979;137(3):332-7.

16. Hirao M, Kurokawa Y, Fujitani K, Tsujinaka T. Randomized controlled trial of Roux-en-Y versus rho-shaped-Roux-en-Y reconstruction after distal gastrectomy for gastric cancer. World $\mathbf{J}$ Surg. 2009;33(2):290-5.

17. NM Mimae T, Nishizaki M, Harano M, Aoki H, Onoda T, Shiozaki S, Oono S, Higaki K, Takakura N. Clinical study of Roux stasis syndrome after distal gastrectomy. Jpn Soc Gastroenterol Surg. 2008;41(8):1551-6.

18. Bertucci W, Yadegar J, Takahashi A, et al. Antecolic laparoscopic Roux-en-Y gastric bypass is not associated with higher complication rates. Am Surg. 2005;71(9):735-7.

19. Murakami Y, Uemura K, Sudo T, et al. An antecolic Roux-en Y type reconstruction decreased delayed gastric emptying after pylorus-preserving pancreatoduodenectomy. J Gastrointest Surg. 2008;12(6):1081-6.

20. Tani M, Terasawa H, Kawai M, et al. Improvement of delayed gastric emptying in pylorus-preserving pancreaticoduodenectomy: results of a prospective, randomized, controlled trial. Ann Surg. 2006;243(3):316-20.

21. Behrns KE, Sarr MG. Diagnosis and management of gastric emptying disorders. Adv Surg. 1994;27:233-55.

22. Cozzaglio L, Coladonato M, Dagrada CT, Doci R, Gennari L. [Does the Roux-en-Y-stasis syndrome still exist?]. Chir Ital. 2005;57(1):27-34.

23. Fraser SA, Shrier I, Miller G, Gordon PH. Immediate postlaparotomy small bowel obstruction: a 16-year retrospective analysis. Am Surg. 2002;68(9):780-2. 